\title{
Missing Galactic PNe: [S III] Imaging Survey
}

\author{
J. Shiode, D. P. Clemens, K. A. Janes and A. Pinnick \\ Institute for Astrophysical Research, Boston Univ., 725 Comm. Ave., Boston, MA, USA
}

\begin{abstract}
The total number of Galactic planetary nebulae (GPNe) is highly uncertain; the most inclusive current catalog contains only $\sim 1,500$. We will use the PRISM wide-field imager on the $1.83 \mathrm{~m}$ Perkins Telescope to conduct a pilot survey of the Galactic plane in search of [S III] emission from PNe obscured by dust and missed by surveys of $\mathrm{H} \alpha$. We are employing the method of Jacoby \& Van de Steene (JVS), who surveyed the bulge for [S III] $\lambda$ 9532. In addition to seeing through more of the extinction, use of the [S III] emission line will a priori reject the most troublesome catalog contaminants: ultracompact H II regions.
\end{abstract}

Keywords. Surveys, Catalogs, Galaxy: disk

Current estimates of the number of Galactic Planetary Nebulae (GPNe) in the Milky Way range from a few thousand (de Marco \& Moe 2005) to several tens of thousands Frew \& Parker (2006). The actual number of observed and verified GPNe is just 3,000 (Parker et al. 2003). Figure 1 is an updated version of the histogram compiled by Kistiakowsky \& Helfand (1993), showing the Galactic latitude distribution of GPNe in the Catalogue of Galactic Planetary Nebulae (Kohoutek 2001). The sample includes all GPNe at low Galactic latitude, and $|\ell|>10^{\circ}$ (to exclude the Galactic bulge). There is a clear deficit in the number of known GPNe near $b=0^{\circ}$, most likely due to obscuration by dark clouds. This low-latitude region is not easy to survey for GPNe using the standard [O III] or $\mathrm{H} \alpha$ signposts because of the large extinctions.

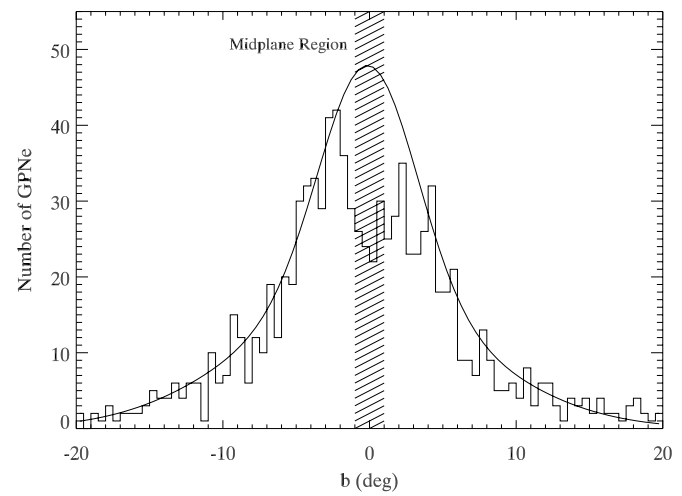

Figure 1. Numbers of known disk PNe binned by Galactic latitude.

The [S III $\lambda$ 9532 emission line is seen in most PNe. Ultra-compact H II regions (UCH II regions) are the most numerous contaminants of current GPNe catalogs. Detectable [S III] $\lambda 9532$ emission is expected from only the hottest and most massive UCH II regions due to the high ionization levels needed to produce and sustain $\mathrm{S}^{++}$. The [S III] $\lambda 9532$ line has been used at least twice before to look for PNe. Kistiakowsky \& Helfand (1993) looked for [S III] $\lambda 9532$ emission from compact $20 \mathrm{~cm}$ radio sources in the Galactic plane, finding [S III] emission toward 10 of 11 candidate PNe, but none associated with candidate SNRs. JVS surveyed the center $4^{\circ} \times 4^{\circ}$ of the Milky Way for obscured PNe, finding 94 new 
candidates - roughly 6 per square degree. Each group found [S III] $\lambda 9532$ to be the most prominent line for $\mathrm{V}$-band extinctions of 4 to $12 \mathrm{mag}$.

Our survey uses two $20 \AA$ narrow bandpass interference filters to sample the emission line and spectrally adjacent continuum. The on-band filter rejects the Paschen 8 recombination line at $9546 \AA$, while avoiding telluric $\mathrm{OH}$ lines. The off-band filter is close in wavelength while also avoiding telluric $\mathrm{OH}$. Two matched filters, placed close together, produce differenced images able to accurately identify [S III] emission sources, and thus candidate GPNe (see Fig. 2)

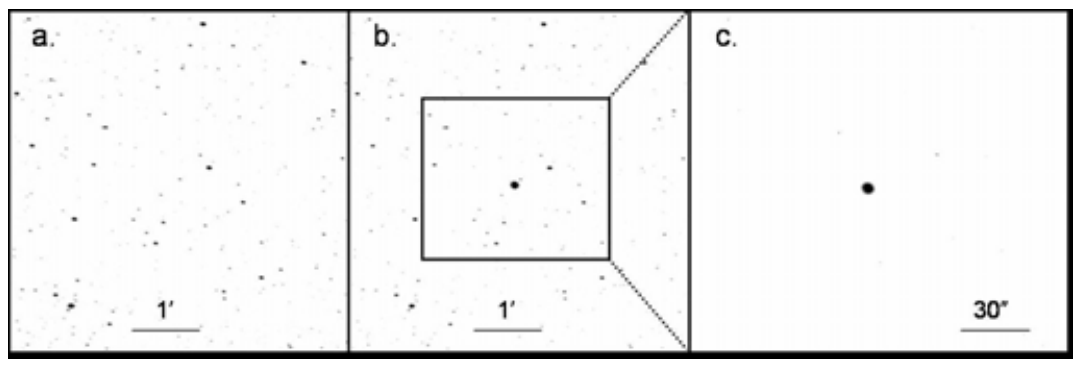

Figure 2. Test field in the Galactic plane, centered on PNG326.7+00.7. a.) off-band image

b.) on-band image c.) an enlarged portion of the on-minus-off differenced image.

Observations will be conducted during Summer (and possibly Fall) 2006 using the new Perkins Re-Imaging System (PRISM) on the Perkins $1.83 \mathrm{~m}$ telescope in Flagstaff, AZ. Figures $2 \mathrm{a}$ and $2 \mathrm{~b}$ display PRISM's $10^{\prime} \times 10^{\prime}$ effective field-of-view. Five minute integrations through each filter for each field allow us to reach the same depth as the JVS survey. We will observe the Galactic plane in one-field-wide latitude strips from $b=-1^{\circ}$ to $b=+1^{\circ}$. Some known GPNe and H II regions will be sampled for calibration.

The images obtained through each filter will be calibrated and on-minus-off differenced images will be computed. We will visually inspect the differenced images in search of resolved [S III] emission, compile point source catalogs with pairs of filter magnitudes, and perform magnitude differencing in search of unresolved [S III] $\lambda 9532$ emission. The resulting list of candidate GPNe will be cross-referenced with current $\mathrm{H} \alpha$ and centimeterwavelength radio surveys for confirmatory evidence about the candidate sources. We expect that verified GPNe selected as candidates using the [S III] $\lambda 9532$ emission line will form a deep, minimally contaminated list of Galactic Planetary Nebulae.

We thank George Jacoby for his help and advice. JS is supported by the NSF-REU Program under grant AST-0440936-1. PRISM was developed with support from the NSF (AST-0079541, KAJ, P. I.), Boston Univ. and Lowell Obs.

\section{References}

de Marco, O. \& Moe, M. 2005, in: Planetary Nebulae as Astronomical Tools AIP Conference Proc. Vol. 804, p. 169

Frew, D. J. \& Parker, Q. A. (2006)

Hanuschik R. W. 2003, A\&̈A 407, 1157

Jacoby, G. H. \& Van de Steene G. 2004, A\&3A 419, 563 (JVS)

Kistiakowsky, V. \& Helfand, D. J. 1993, ApJ 105, 2199

Kohoutek, L. 2001, A\&A 378, 843

Parker, Q. A., Hartley, M., Russeil, D., et al. 2003, in: S. Kwok, M. Dopita \& R. Sutherland (eds.), Proc. IAU Symposium No. 209, p. 25 